Hadron Structure '15

International Journal of Modern Physics: Conference Series

Vol. 39 (2015) 1560081 (14 pages)

(C) The Author(s)

DOI: $10.1142 / \mathrm{S} 2010194515600812$

\title{
Light-front holography and superconformal quantum mechanics: A new approach to hadron structure and color confinement
}

\author{
Stanley J. Brodsky \\ SLAC National Accelerator Laboratory, Stanford University, \\ Stanford, California 94039, USA \\ sjbth@slac.stanford.edu \\ Alexandre Deur \\ Thomas Jefferson National Accelerator Facility, \\ Newport News, VA 23606, USA \\ deurpam@jlab.org \\ Guy F. de Téramond \\ Universidad de Costa Rica, San José, Costa Rica \\ gdt@asterix.crnet.cr \\ Hans Günter Dosch \\ Institut für Theoretische Physik, Philosophenweg 16, \\ D-6900 Heidelberg, Germany \\ h.g.dosch@thphys.uni-heidelberg.de
}

Published 26 November 2015

A primary question in hadron physics is how the mass scale for hadrons consisting of light quarks, such as the proton, emerges from the QCD Lagrangian even in the limit of zero quark mass. If one requires the effective action which underlies the QCD Lagrangian to remain conformally invariant and extends the formalism of de Alfaro, Fubini and Furlan to light-front Hamiltonian theory, then a unique, color-confining potential with a mass parameter $\kappa$ emerges. The actual value of the parameter $\kappa$ is not set by the model - only ratios of hadron masses and other hadronic mass scales are predicted. The result is a nonperturbative, relativistic light-front quantum mechanical wave equation, the Light-Front Schrödinger Equation which incorporates color confinement and other essential spectroscopic and dynamical features of hadron physics, including a massless pion for zero quark mass and linear Regge trajectories with the identical slope in the radial quantum number $n$ and orbital angular momentum $L$. The same light-front equations for mesons with spin $J$ also can be derived from the holographic mapping to $\mathrm{QCD}(3+1)$ at fixed light-front time from the soft-wall model modification of $\mathrm{AdS}_{5}$

This is an Open Access article published by World Scientific Publishing Company. It is distributed under the terms of the Creative Commons Attribution 3.0 (CC-BY) License. Further distribution of this work is permitted, provided the original work is properly cited. 


\section{S. J. Brodsky et al.}

space with a specific dilaton profile. Light-front holography thus provides a precise relation between the bound-state amplitudes in the fifth dimension of AdS space and the boost-invariant light-front wavefunctions describing the internal structure of hadrons in physical space-time. One can also extend the analysis to baryons using superconformal algebra $-2 \times 2$ supersymmetric representations of the conformal group. The resulting fermionic LF bound-state equations predict striking similarities between the meson and baryon spectra. In fact, the holographic QCD light-front Hamiltonians for the states on the meson and baryon trajectories are identical if one shifts the internal angular momenta of the meson $\left(L_{M}\right)$ and baryon $\left(L_{B}\right)$ by one unit: $L_{M}=L_{B}+1$. We also show how the mass scale $\kappa$ underlying confinement and the masses of light-quark hadrons determines the scale $\Lambda_{\overline{M S}}$ controlling the evolution of the perturbative QCD coupling. The relation between scales is obtained by matching the nonperturbative dynamics, as described by an effective conformal theory mapped to the light-front and its embedding in AdS space, to the perturbative QCD regime. The data for the effective coupling defined from the Bjorken sum rule $\alpha_{g_{1}}\left(Q^{2}\right)$ are remarkably consistent with the Gaussian form predicted by LF holographic QCD. The result is an effective coupling defined at all momenta. The predicted value $\Lambda_{\frac{\left(N_{F}\right.}{M S}}=0.440 m_{\rho}=0.341 \pm 0.024 \mathrm{GeV}$ is in agreement with the world average $0.339 \pm 0.010 \mathrm{GeV}$. We thus can connect $\Lambda_{\overline{M S}}$ to hadron masses. The analysis applies to any renormalization scheme.

\section{Emergence of the QCD Mass Scale}

A central feature of QCD for zero quark mass is the appearance of the hadronic mass scale, even though no parameter with mass dimension appears explicitly in the QCD Lagrangian. In fact, at the semiclassical level, QCD with massless quarks is conformally invariant.

The emergence of a mass scale in an underlying conformal theory was investigated in the context of one-dimensional quantum mechanics in 1976 in a pioneering paper by de Alfaro, Fubini, and Furlan (dAFF). ${ }^{1}$ They showed that one can modify the Hamiltonian of a conformal theory with a new term containing a constant $\kappa$ with the dimension of mass while retaining the conformal invariance of the action by including contributions proportional to the dilatation and special conformal operators of the conformal group. The result is a new term in the Hamiltonian which has the unique form of a confining harmonic oscillator potential. Thus not only does a mass scale emerge, but the spectrum only consists of bound states. Remarkably, the action remains conformally invariant if one transforms the time variable from ordinary time $t$ to a new evolution parameter $\tau$ which can be interpreted as the light-front time $t+z / c$, the time along a light-front (LF). The range of $\tau$ is finite reflecting the finite difference of light-front times between the confined constituents. ${ }^{2}$

The dAFF procedure can be applied to hadron physics ${ }^{2,3}$ in physical $(3+1)$ space-time in the context of light-front quantization; i.e., Dirac's "Front Form". One can define the LF Hamiltonian $H_{L F}=-i \frac{d}{d \tau}$ directly from the QCD Lagrangian. The eigensolutions of the LF Hamlitonian predict the hadronic mass spectrum and the frame-independent light-front wavefunctions which underly hadronic observables form factors, structure functions, transverse momentum distributions, etc. As shown in Fig. 1, the exact LF Heisenberg equation $H_{L F} \Psi=M^{2} \mid \Psi>$ for mesons can be systematically reduced to an effective LF Schrödinger Equation (LFSE) operating 


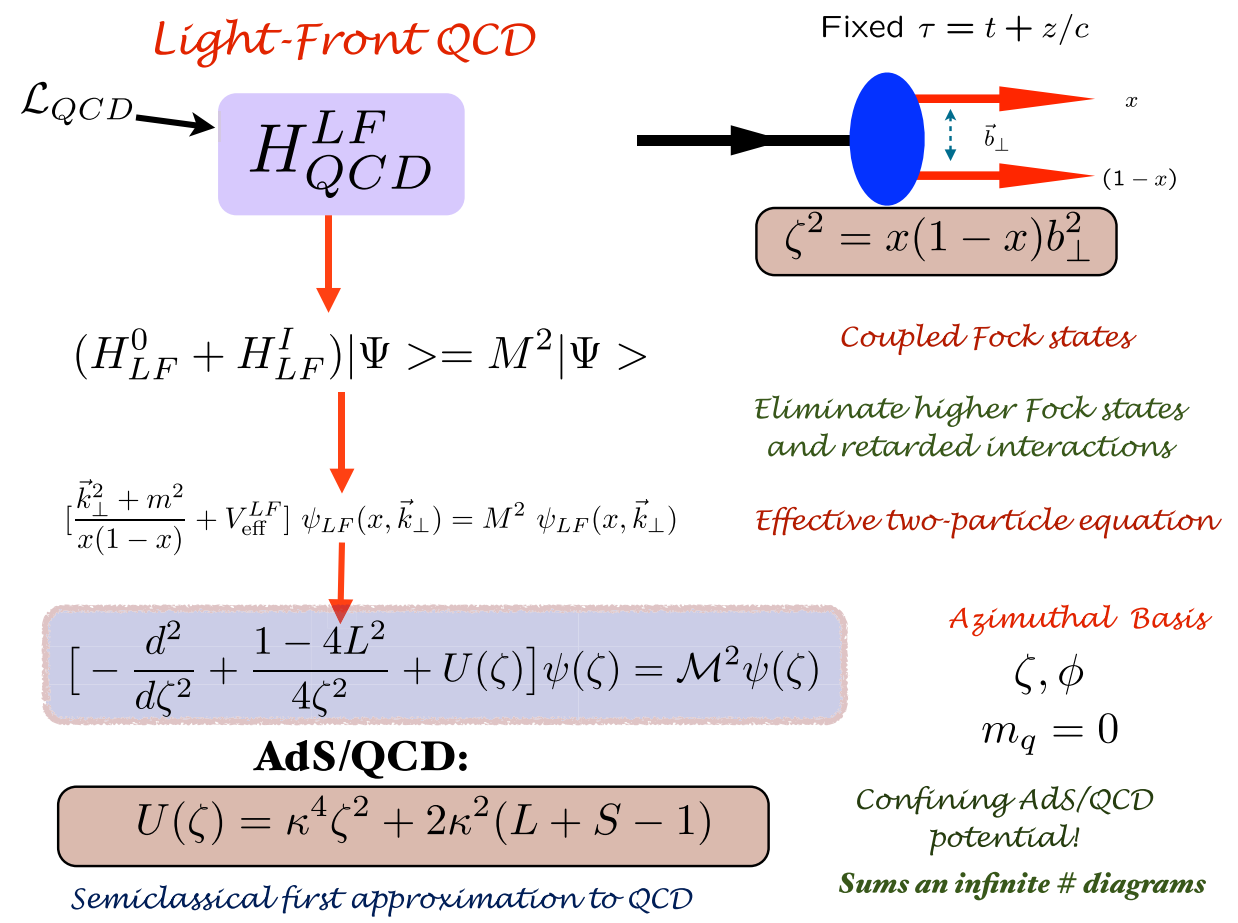

Fig. 1. Systematic reduction of the full LF Hamiltonian of QCD to an effective LF Schrödinger equation for the $q \bar{q}$ valence meson Fock state.

on the lowest $q \bar{q}$ Fock state. (In the case of QED, similar steps generate the effective bound state equation for hydrogenic atoms, including the Lamb Shift and hyperfine splitting.) The resulting LF equation is frame-independent.

If one projects on valence states of fixed $L^{z}$, the result is a one dimensional equation in the LF radial variable $\zeta$ where $\zeta^{2}=b_{\perp}^{2} x(1-x)$ is conjugate to the $q \bar{q}$ LF kinetic energy $\frac{k_{\perp}^{2}}{x(1-x)}$, which is also the square of the $q \bar{q}$ invariant mass $\mathcal{M}_{q \bar{q}}^{2}=\left(p_{q}+p_{\bar{q}}\right)^{2}$ for massless quarks

$$
\left[-\frac{d^{2}}{d \zeta^{2}}+\frac{1-4 L^{2}}{\zeta^{2}}+U\left(\zeta^{2}\right)\right] \Psi_{q \bar{q}}(\zeta)=M_{H}^{2} \Psi_{q \bar{q}}(\zeta)
$$

where $L=\max \left|L^{z}\right|$. The confining potential $U(\zeta)^{2}$ in this effective two-body equation reflects not only the usual QCD interactions, but also the effects from the elimination of the higher Fock states. However, following the procedure of dAFF, the LF potential can only have form $U(\zeta)^{2}=\kappa^{4} \zeta^{2}+$ const, if one demands that the action of the semiclassical theory remain conformally invariant. This procedure not only introduces the hadronic mass scale $\kappa$ but it also ensures that only confined colored states can emerge. The masses of each hadron consisting of light quarks and features such as decay constants and the inverse of the slope of hadronic form 


\section{S. J. Brodsky et al.}

factors will also scale with $\kappa$. It should be emphasized that the absolute value of $\kappa$ in units such as $\mathrm{MeV}$ is not determined by chiral QCD - only dimensionless ratios are predicted by the theory. Thus in a sense, the model has no parameters.

Remarkably, the identical bound state equations appear in the formalism built on $\mathrm{AdS}_{5}{ }^{4}$ - the five-dimensional representation of the isometries of the conformal group. In fact, because of light-front holography, ${ }^{5}$ the equations derived from the $\mathrm{AdS}_{5}$ action are identical to LF equations in $3+1$ space-time at fixed LF time $\tau$ when one identifies the fifth dimension coordinate $z$ of $\mathrm{AdS}_{5}$ space with the LF transverse coordinate $\zeta$. The Polchinski-Strassler formulae for electromagnetic and gravitational form factors in $\mathrm{AdS}_{5}$ coincide with the Drell-Yan West LF formulae, an important feature of light-front holography. ${ }^{6}$ The identical potential emerges: $U(\zeta)^{2}=\kappa^{4} \zeta^{2}$ together with a $J$-dependent constant $2 \kappa^{2}(J-1)$ for spin- $J$ meson representations ${ }^{7}$ if one modifies the $\mathrm{AdS}_{5}$ action by the "soft-wall" dilaton factor $e^{+\kappa^{2} z^{2}}$. Thus the combination of the soft wall AdS/QCD model, light-front holography, and the dAFF procedure for retaining the conformal invariance of the action in LF Hamiltonian theory leads to the LF Schrödinger equation with the emerging scale $\kappa$ illustrated Fig. 1. The eigenvalues of this LF Hamiltonian give a simple form for the meson spectrum for massless quarks: $M^{2}(n, L, J)=4 \kappa^{2}\left(n+\frac{L+J}{2}\right)=4 \kappa^{2}\left(n+L+\frac{S}{2}\right)$. A comparison of the predicted spectrum with the observed $J=0$ and $J=1$ meson trajectories consisting of $u, d, s$ quarks is shown in Fig. 2. The predictions for hadrons containing strange quarks are obtained by including the quark mass term $\sum_{i} \frac{m_{i}^{2}}{x_{i}}$ in the LF kinetic energy.

\section{Features of the LF Schrödinger Equation}

The LF Schrödinger Equation has a number of remarkable features for both hadronic spectroscopy and dynamics.

- The pion eigenstate $(n=L=J=0)$ is massless for zero quark mass. The positive terms from the LF kinetic energy and the confining LF harmonic oscillator potential are precisely cancelled by the negative constant term. The physical pion mass corresponds to $m_{q}=54 \mathrm{MeV}$.

- The predicted Regge trajectories are linear in $n$ and $L$ with the identical slope: $\mathcal{M}^{2}=4 \kappa^{2}(n+L+S / 2)$.

- The $\mathcal{M}^{2}$ of the $S=1$ vector meson and $S=0$ pseudoscalar trajectories are separated by $2 \kappa^{2}$ for all $n$ and $L$.

- The eigenfunctions determine the hadronic LF wavefunctions $\psi\left(x, k_{\perp}\right)$ in the nonperturbative domain which in turn determine hadron form factors, structure functions, etc. The transverse momentum dependence $k_{\perp}$ always appears in the rotationally invariant combination $k_{\perp}^{2} / x(1-x) .{ }^{8}$ See Fig. 3 .

- The predicted LF wavefunction of the $\rho$ meson provides an excellent description of the empirical features of $\rho$ electroproduction as shown by Forshaw and Sandapen. ${ }^{9}$ 

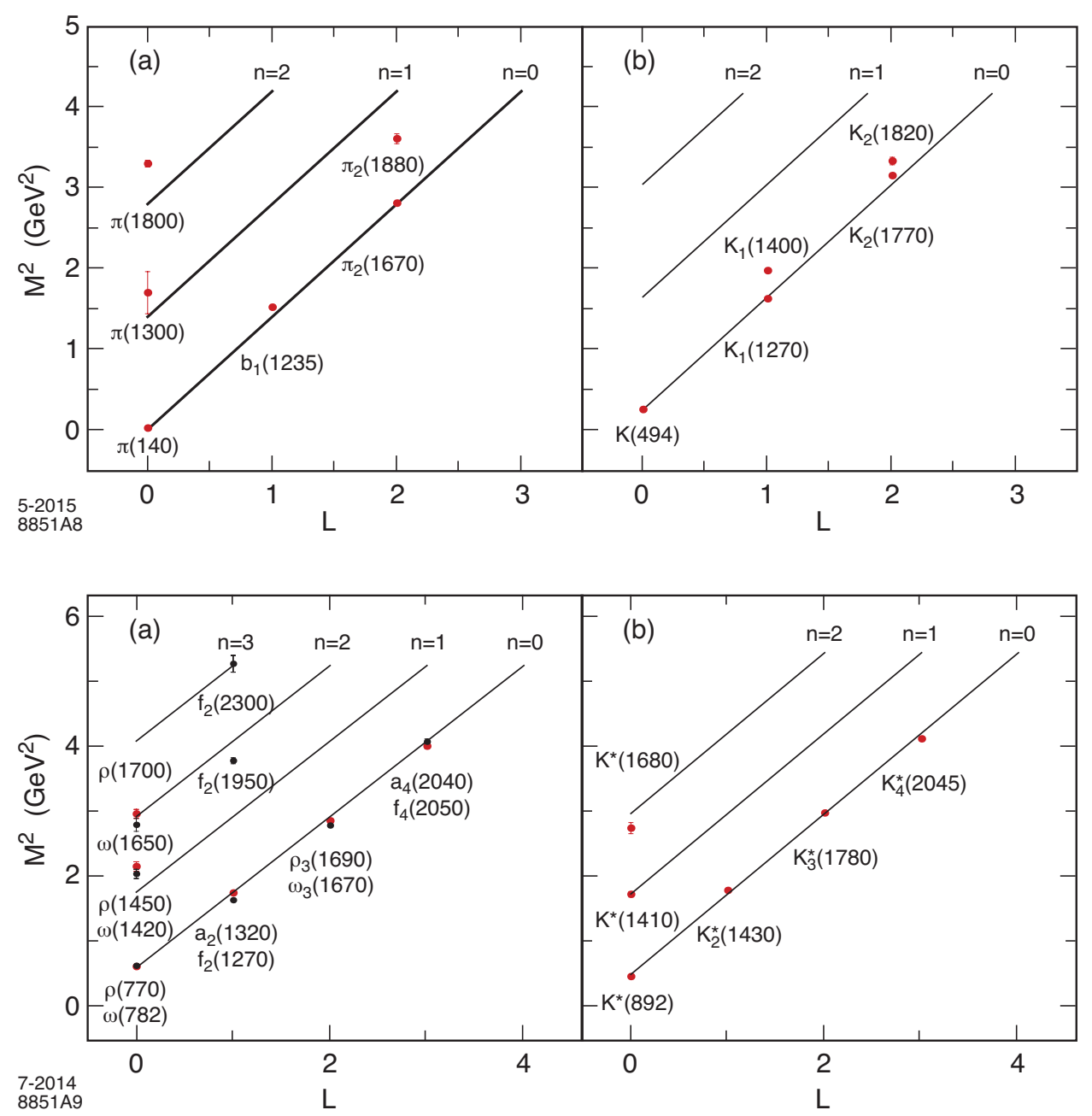

Fig. 2. The predicted $S=0$ and $S=1$ meson trajectories in $n$ and $L$ from LF holography.

- The predicted form for the pion distribution amplitude $\phi_{\pi}(x)=\frac{4 f_{\pi}}{\sqrt{3}} \sqrt{x(1-x)}$ in the nonperturbative domain leads to a prediction for the photon-to-pion transition form factor in agreement with Belle data. ${ }^{10}$ The $\sqrt{x(1-x)}$ dependence agrees with Dyson-Schwinger determinations. ${ }^{11}$ It evolves to the $x(1-x)$ asymptotic form ${ }^{12,13}$ as $\log Q^{2} \rightarrow \infty$ using ERBL evolution.

- Although the potential between light quarks is a harmonic oscillator, the corresponding nonrelativistic potential $V(r)$ between heavy quarks is linear in $r$, consistent with quarkonium phenomenology. This connection ${ }^{14}$ is due to the fact that the eigenvalue in $\mathcal{M}^{2}$ is effectively the square of the energy eigenvalue in the $\vec{P}=0$ rest frame. 


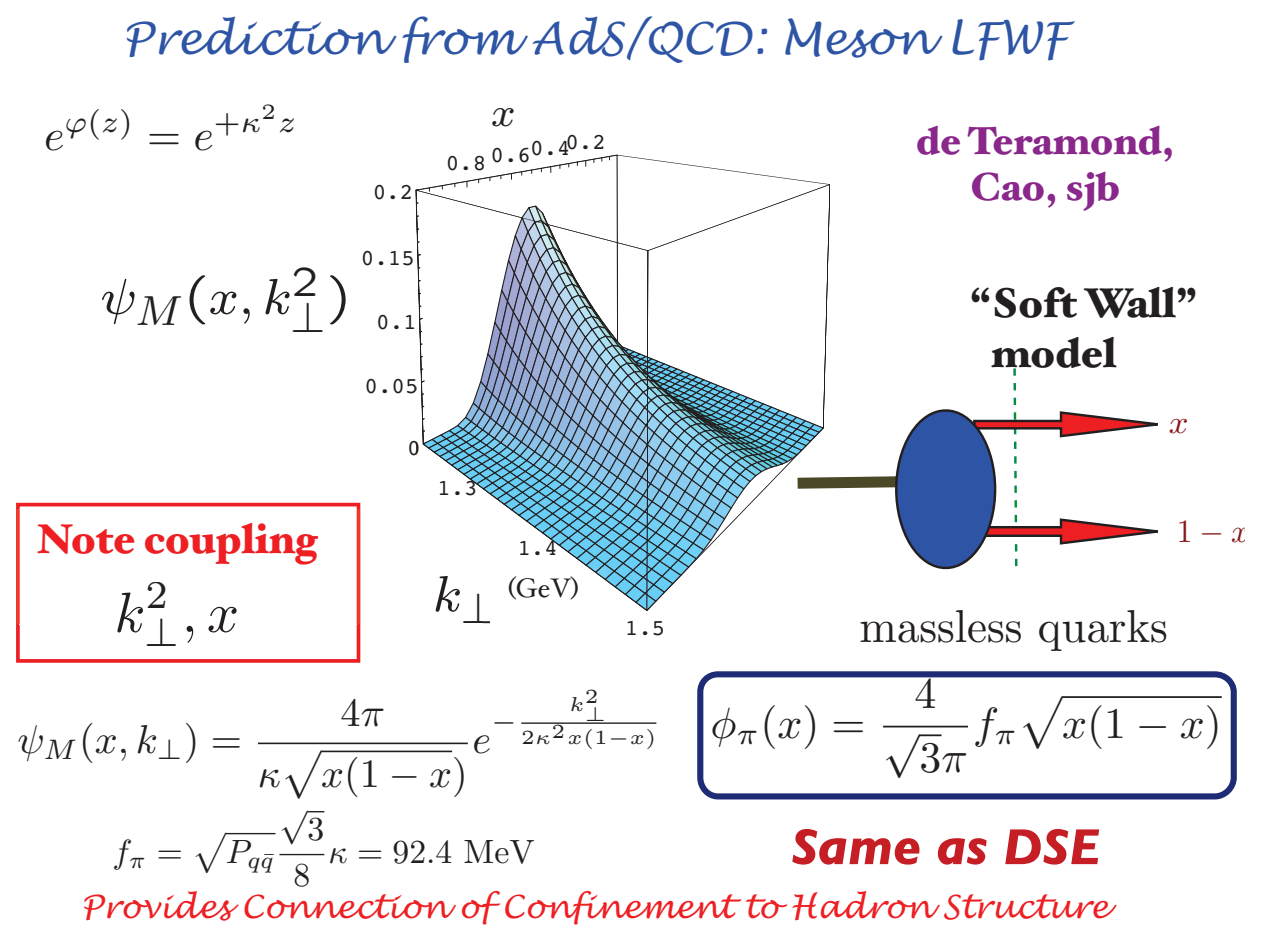

Fig. 3. The LF wavefunctions and distribution amplitudes of mesons predicted by the LF Schrödinger Equation.

\section{Superconformal Algebra and Supersymmetric Features of QCD}

The dAFF procedure can be generalized to baryon states $^{15}$ for massless quarks using the $2 \times 2$ Pauli matrix representation of the conformal group. ${ }^{16}$ The potential in the LF equations are generated by the superconformal generator $S$ where the special conformal operator $K=\left\{S, S^{\dagger}\right\}$. The resulting two-component LF equations describe baryons as a quark plus a scalar diquark with the quark $S_{q}^{z}$ parallel or anti-parallel to the parent baryon's $S_{B}^{z}$, as well as the LFSE for mesons. See Fig. 4.

The eigensolutions have the remarkable feature that the entire baryon and meson spectra are degenerate if $L_{M}=L_{B}+1$; i.e. the mesons and baryons are superpartners if one makes a shift of the internal angular momentum of the mesons by one unit of orbital angular momentum. See Fig. 5. The pion and other mesons with $L=0$ have no superpartners. The empirical baryon and meson trajectories have the same slope in $n$ and $L$, as predicted by holographic QCD. In fact, the best fits to the numerical values for the Regge slopes agree within $\pm 10 \%$ for all hadrons, mesons and baryons; this leads to a near-degeneracy of the meson and baryon levels in the model. 
Fig. 4. The effective LF bound state equations for baryons and mesons with $m_{q}=0$ derived from superconformal algebra. The LF wavefunctions $\psi_{J}^{ \pm}$refer to the quark spin $S_{q}^{z}$ parallel or antiparallel to the parent baryon spin $S_{B}^{z}$. The two amplitudes have the same mass squared eigenvalues and equal probabilities.

The nucleon form factors predicted by this formalism are shown in Fig. 6. Since the LF wavefunctions of mesons and baryons with $L_{M}=L_{B}+1$ and have same mass and twist, one expects that their respective form factors will also be identical.

A generalization to heavy quark states is given in Ref.. ${ }^{17}$ The idea to apply supersymmetry to hadron physics is not new. ${ }^{18-20} \mathrm{In}^{18}$ mesons and baryons are grouped together in a large supermultiplet, a representation of $U_{6 / 21}$. In Ref. 19 the supersymmetry results of Miyazawa ${ }^{18}$ are recovered in a QCD framework, provided that a diquark configuration emerges through an effective string interaction.

\section{The Non-Perturbative QCD Coupling}

As noted above, the color confining potential of the LF Hamiltonian can be derived from the modification of the AdS space curvature, the soft-wall dilaton profile ${ }^{2}$ $e^{+\kappa^{2} z^{2}}$ which is determined by the form of the potential dictated by the dAFF mechanism. This same profile leads to an explicit form for the QCD coupling at long distances using LF holography. ${ }^{21-23}$ We will apply the AdS/QCD prediction to $\alpha_{g_{1}}^{A d S}\left(Q^{2}\right)$, the effective charge in the $g_{1}$-scheme ${ }^{24}$ defined from the Bjorken sum 


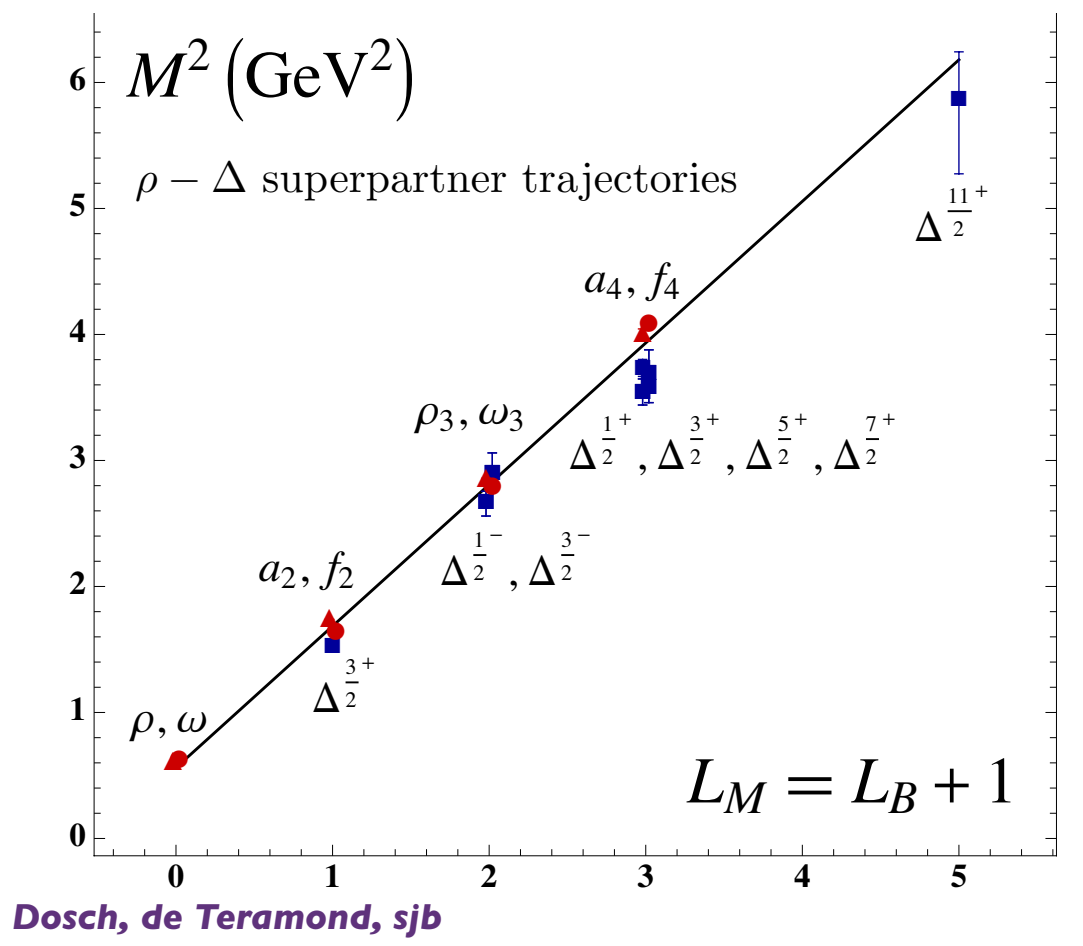

Fig. 5. A comparison of meson and baryon Regge trajectories.

rule. ${ }^{25,26}$ It is normalized to $\pi$ at $Q^{2}=0$ due to kinematical constraints. ${ }^{27,28}$ This well-measured coupling can serve as the QCD-analog of the Gell-Mann-Low coupling $\alpha\left(Q^{2}\right)$ of QED. ${ }^{21}$

The 5-dimensional AdS action is

$$
S=\frac{1}{4} \int d^{5} x \sqrt{\operatorname{det}\left(g_{\mu \nu}^{A d S}\right)} e^{\kappa^{2} z^{2}} \frac{1}{g_{A d S}^{2}} F^{2} .
$$

The initially constant AdS coupling $\alpha_{A d S} \equiv g_{A d S}^{2} / 4 \pi$ can be redefined to absorb the effects of the AdS deformation which determines the long-range confining interaction. It predicts the form: $g_{A d S}^{2} \rightarrow g_{A d S}^{2} e^{\kappa^{2} z^{2}}$. This constraint also controls the form of $\alpha_{s}$ at small $Q^{2}$. Transforming to momentum space yields ${ }^{21}$

$$
\alpha_{g_{1}}^{A d S}\left(Q^{2}\right)=\pi e^{-Q^{2} / 4 \kappa^{2}} .
$$

In pQCD, $\alpha_{s} \equiv g_{s}^{2} / 4 \pi$ acquires its large $Q^{2}$-dependence from short-distance quantum effects. It is given by the QCD renormalization group equation, where the logarithmic derivative of the coupling defines the $\beta$ function. If $\alpha_{s}$ is small, one can use the perturbative expansion

$$
Q^{2} d \alpha_{s} / d Q^{2}=-\left(\beta_{0} \alpha_{s}^{2}+\beta_{1} \alpha_{s}^{3}+\beta_{2} \alpha_{s}^{4}+\cdots\right) .
$$


Using $S U(6)$ flavor symmetry and normalization to static quantities
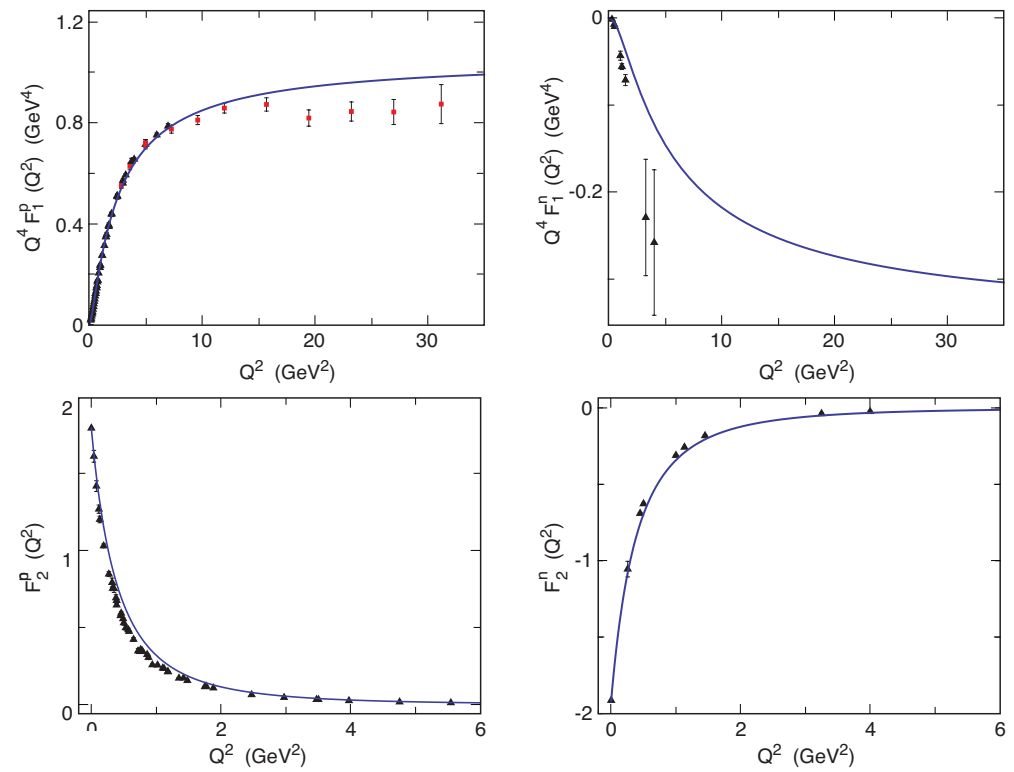

Fig. 6. Predictions for the nucleon Dirac and Pauli form factors. The Pauli form factors are normalized to the nucleon anomalous magnetic moments.

The $\beta_{i}$ for $i \geq 2$ are scheme-dependent and are known up to order $\beta_{3}$ in the $\overline{M S}$ renormalization scheme. Eq. (4) thus yields $\alpha_{\overline{M S}}\left(Q^{2}\right)$ at high $Q^{2}$. In addition, $\alpha_{g_{1}}^{p Q C D}\left(Q^{2}\right)$ can be expressed as a perturbative expansion in $\alpha_{\overline{M S}}\left(Q^{2}\right){ }^{25,26}$ Thus, pQCD predicts the form of $\alpha_{g_{1}}\left(Q^{2}\right)$ with asymptotic freedom at large $Q^{2}$.

The existence at moderate values of $Q^{2}$ of a dual description of QCD in terms of either quarks and gluons versus hadrons ("quark-hadron duality" ${ }^{29}$ ) is consistent with the matching of $\alpha_{g_{1}}^{p Q C D}$ to $\alpha_{g_{1}}^{A d S}$ at intermediate values of $Q^{2}$.

This matching can be done by imposing continuity of both $\alpha_{g_{1}}\left(Q^{2}\right)$ and its first derivative, as shown in Fig. 7. The unique solution for the resulting two equalities determines the scale parameter of the running coupling $\Lambda_{\overline{M S}}$ from $\kappa$, and fixes the scale $Q_{0}$ characterizing the transition between the large and short-distance regimes of QCD. The result at order $\beta_{3}$, the same order to which the experimental value of $\Lambda_{\overline{M S}}$ is extracted, is $\Lambda_{\overline{M S}}=0.341 \pm 0.024 \mathrm{GeV}$. The uncertainty stems from the extraction of $\kappa$ from the $\rho$ or proton masses and from a small contribution from ignoring the quark masses. This theory uncertainty is less or comparable to that of the experimental determinations, which combine to ${ }^{22} \Lambda_{\overline{M S}}=0.339 \pm 0.016 \mathrm{GeV}$.

We thus can determine $\Lambda_{\overline{M S}}$ from hadron masses: $\Lambda_{\overline{M S}}=0.440 m_{\rho}=0.622 \kappa$. The scale $Q_{0}^{2}=1.25 \mathrm{GeV}^{2}$ determines the transition between the non-perturbative and perturbative domains. It can serve as the starting point for DGLAP and ERBL evolution in the $\overline{M S}$ scheme. 


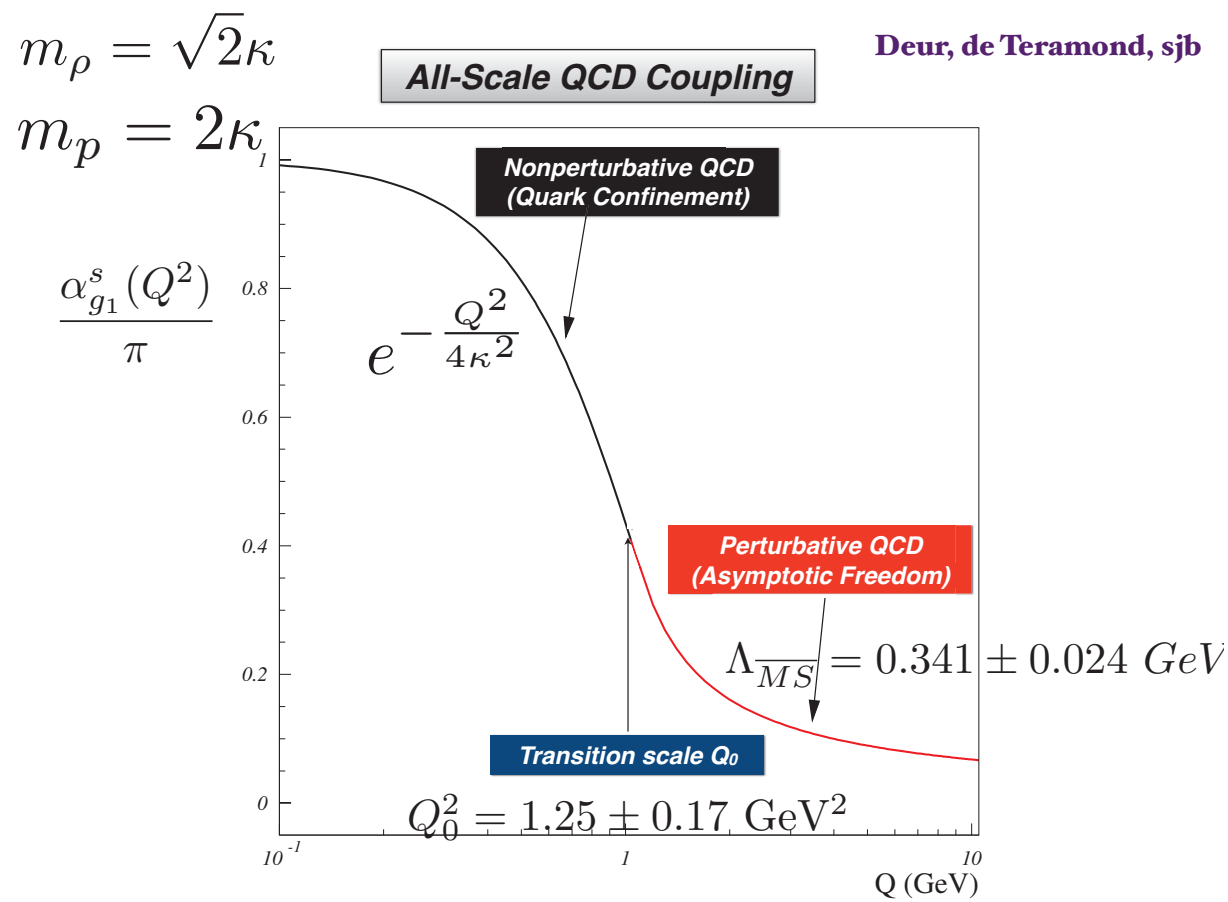

Fig. 7. The strong coupling $\alpha_{g 1}\left(Q^{2}\right)$ obtained from the analytic matching of perturbative and non-perturbative QCD regimes.

In Fig. 8, the AdS/QCD prediction for $\alpha_{g_{1}}^{A d S}\left(Q^{2}\right)(3)$ is plotted together with data. $^{27,28}$ Even though there are no adjustable parameters, the predicted Gaussian form for the behavior of $\alpha_{g_{1}}^{A d S}\left(Q^{2}\right)$ at $Q^{2}<Q_{0}^{2}$ agrees well with data. ${ }^{21}$ There is little dependence of $\alpha_{g_{1}}^{p Q C D}\left(Q^{2}\right)$ on the order of perturbation theory in $\beta_{n}$ and $\alpha \frac{n}{M S}$.

\section{Hadronization at the Amplitude Level and Other New Directions}

- The new insights into color confinement given by AdS/QCD suggest that one could compute hadronization at amplitude level ${ }^{30}$ using LF time-ordered perturbation theory, but including the confinement interaction. For example, if one computes $e^{+} e^{-} \rightarrow q \bar{q} \rightarrow q \bar{q} g \cdots$, the quarks and gluons only appear in intermediate states, and only hadrons can be produced. LF perturbation theory provides a remarkably efficient method for the calculation of multi-gluon amplitudes. ${ }^{31}$

- The eigensolutions of the AdS/QCD LF Hamiltonian can used to form an orthonormal basis for diagonalizing the complete QCD LF Hamiltonian. This method, "basis light-front quantization" 32 is expected to be more efficient than the DLCQ $\operatorname{method}^{33}$ for obtaining QCD $3+1$ solutions. 


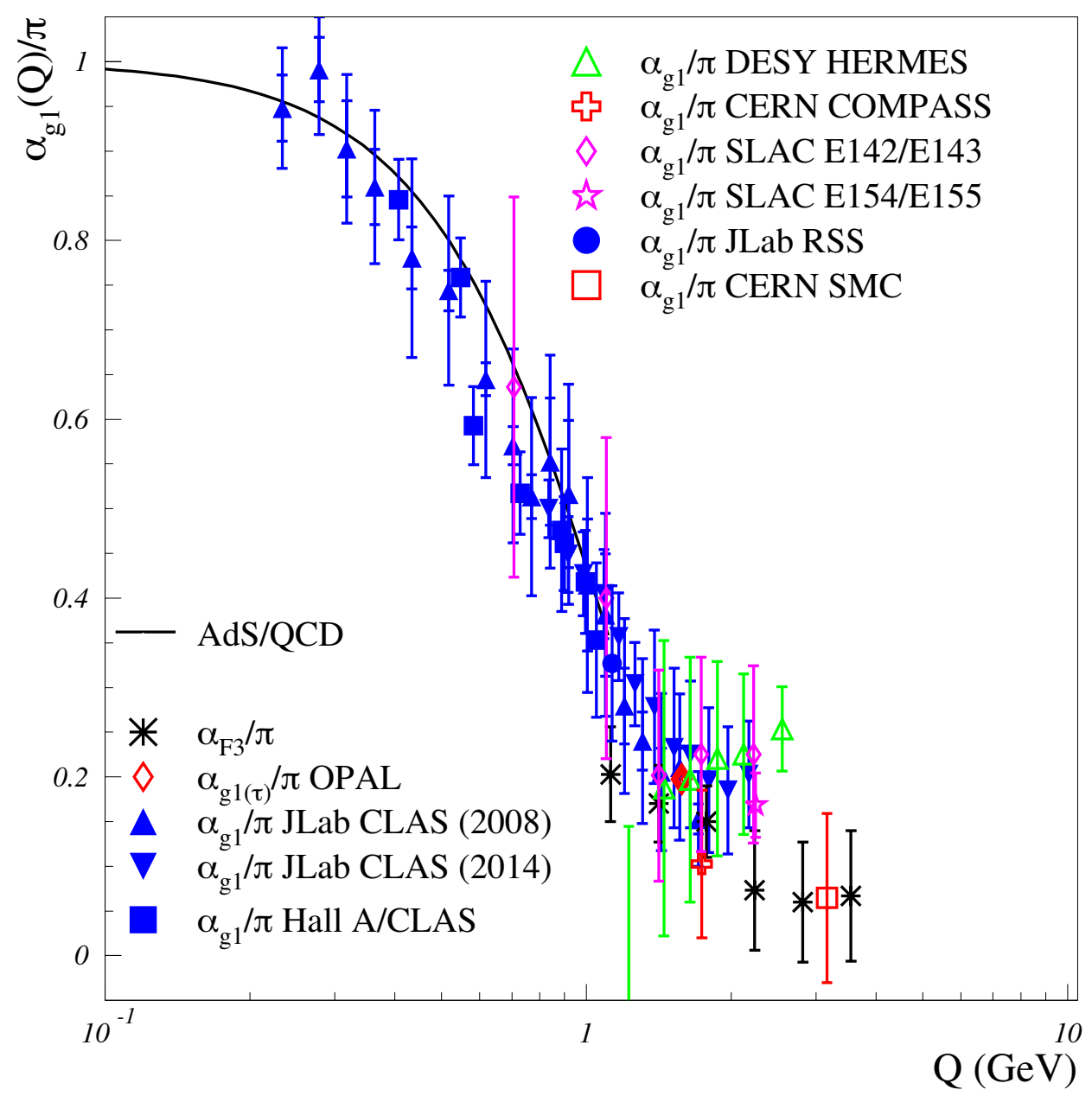

Fig. 8. The analytic non-perturbative AdS/QCD prediction $\alpha_{g_{1}}^{A d S}\left(Q^{2}\right)=\pi e^{-Q^{2} / 4 \kappa^{2}}$ compared with measurements and the Bjorken sum rule. The data are plotted in the domain between $0<$ $Q<Q_{0}$ where AdS/QCD is a valid approximation.

- All of the hadron physics predictions discussed in this report are independent of the value of $\kappa$; only dimensionless ratios are predicted, such as $m_{p}=\sqrt{2} m_{\rho}$ and the ratio $\Lambda_{\bar{M} S} / m_{\rho}$. The ratio can be obtained in any renormalization scheme. One thus retains dilatation invariance $\kappa \rightarrow \gamma \kappa$ of the prediction.

- The $\kappa^{4} \zeta^{2}$ confinement interaction between a $q$ and $\bar{q}$ will induce a $\kappa^{4} / s^{2}$ correction to $R_{e^{+} e^{-}}$, replacing the $1 / s^{2}$ signal usually attributed to a vacuum gluon condensate.

- The kinematic condition that all $k^{+}=k^{0}+k^{3}$ are positive and conserved precludes QCD condensate contributions to the $P^{+}=0 \mathrm{LF}$ vacuum state, which by definition is the causal, frame-independent lowest invariant mass eigenstate of the LF Hamiltonian. ${ }^{34,35}$ 


\section{S. J. Brodsky et al.}

- It is interesting to note that the contribution of the ' $H$ ' diagram to $Q \bar{Q}$ scattering is IR divergent as the transverse separation between the $Q$ and the $\bar{Q}$ increases. ${ }^{36}$ This is a signal that pQCD is inconsistent without color confinement. The sum of such diagrams could sum to the confinement potential $\kappa^{4} \zeta^{2}$ dictated by the dAFF principle that the action remains conformally invariant despite the mass scale in the Hamiltonian.

\section{Acknowledgments}

Presented by SJB at Hadron Structure 15, Horny Smokovec, Slovak Republic, June 26 to July 3, 2015. We also thank Cecil Lorce and Kelly Yu-Ju Chiu for helpful conversations. This research was supported by the Department of Energy, contract DE-AC02-76SF00515. SLAC-PUB-16397.

\section{References}

1. V. de Alfaro, S. Fubini and G. Furlan, "Conformal Invariance in Quantum Mechanics," Nuovo Cim. A 34, 569 (1976).

2. S. J. Brodsky, G. F. De Teramond and H. G. Dosch, "Threefold Complementary Approach to Holographic QCD," Phys. Lett. B 729, 3 (2014), [arXiv:1302.4105 [hepth]].

3. H. G. Dosch, S. J. Brodsky and G. F. de Teramond, "Modified Anti-de-Sitter Metric, Light-Front Quantized QCD, and Conformal Quantum Mechanics," arXiv:1401.7710 [hep-ph].

4. S. J. Brodsky, G. F. de Teramond, H. G. Dosch and J. Erlich, "Light-Front Holographic QCD and Emerging Confinement," Phys. Rept. 584, 1 (2015), [arXiv:1407.8131 [hep$\mathrm{ph}]$.

5. S. J. Brodsky and G. F. de Teramond, "Light-front hadron dynamics and AdS/CFT correspondence," Phys. Lett. B 582, 211 (2004), [hep-th/0310227].

6. S. J. Brodsky and G. F. de Teramond, "Light-Front Dynamics and AdS/QCD Correspondence: The Pion Form Factor in the Space- and Time-Like Regions," Phys. Rev. D 77, 056007 (2008), [arXiv:0707.3859 [hep-ph]].

7. G. F. de Teramond, H. G. Dosch and S. J. Brodsky, "Kinematical and Dynamical Aspects of Higher-Spin Bound-State Equations in Holographic QCD," Phys. Rev. D 87, no. 7, 075005 (2013), [arXiv:1301.1651 [hep-ph]].

8. S. J. Brodsky, T. Huang and G. P. Lepage, "Hadronic and nuclear interactions in QCD," Springer Tracts Mod. Phys. 100, 81 (1982).

9. J. R. Forshaw and R. Sandapen, "An AdS/QCD holographic wavefunction for the rho meson and diffractive rho meson electroproduction," Phys. Rev. Lett. 109, 081601 (2012), [arXiv:1203.6088 [hep-ph]].

10. S. J. Brodsky, F. G. Cao and G. F. de Teramond, "Meson Transition Form Factors in Light-Front Holographic QCD," Phys. Rev. D 84, 075012 (2011), [arXiv:1105.3999 [hep-ph]].

11. L. Chang, C. Mezrag, H. Moutarde, C. D. Roberts, J. Rodrguez-Quintero and P. C. Tandy, "Basic features of the pion valence-quark distribution function," Phys. Lett. B 737, 23 (2014), [arXiv:1406.5450 [nucl-th]].

12. G. P. Lepage and S. J. Brodsky, "Exclusive Processes in Quantum Chromodynamics: Evolution Equations for Hadronic Wave Functions and the Form-Factors of Mesons," Phys. Lett. B 87, 359 (1979). 
13. G. P. Lepage and S. J. Brodsky, "Exclusive Processes in Perturbative Quantum Chromodynamics," Phys. Rev. D 22, 2157 (1980).

14. A. P. Trawinski, S. D. Glazek, S. J. Brodsky, G. F. de Teramond and H. G. Dosch, "Effective confining potentials for QCD," Phys. Rev. D 90, no. 7, 074017 (2014), [arXiv:1403.5651 [hep-ph]].

15. G. F. de Teramond, H. G. Dosch and S. J. Brodsky, "Baryon Spectrum from Superconformal Quantum Mechanics and its Light-Front Holographic Embedding," Phys. Rev. D 91, no. 4, 045040 (2015), [arXiv:1411.5243 [hep-ph]].

16. S. Fubini and E. Rabinovici, "Superconformal Quantum Mechanics," Nucl. Phys. B 245, 17 (1984).

17. H. G. Dosch, G. F. de Teramond and S. J. Brodsky, "Supersymmetry Across the Light and Heavy-Light Hadronic Spectrum," arXiv:1504.05112 [hep-ph].

18. H. Miyazawa, "Baryon Number Changing Currents," Prog. Theor. Phys. 36, no. 6, 1266 (1966).

19. S. Catto and F. Gursey, "Algebraic Treatment of Effective Supersymmetry," Nuovo Cim. A 86, 201 (1985).

20. D. B. Lichtenberg, "Whither hadron supersymmetry?," hep-ph/9912280.

21. S. J. Brodsky, G. F. de Teramond and A. Deur, "Nonperturbative QCD Coupling and its $\beta$-function from Light-Front Holography," Phys. Rev. D 81, 096010 (2010), [arXiv:1002.3948 [hep-ph]].

22. A. Deur, S. J. Brodsky and G. F. de Teramond, "The Relation between the Perturbative QCD Scale $\Lambda_{s}$ and Hadronic Masses from Light-Front Holography," arXiv:1509.03112 [hep-ph].

23. S. J. Brodsky, G. F. de Teramond, A. Deur and H. G. Dosch, "The Light-Front Schrdinger Equation and the Determination of the Perturbative QCD Scale from Color Confinement: A First Approximation to QCD," Few Body Syst. 56, no. 6-9, 621 (2015), [arXiv:1410.0425 [hep-ph]].

24. G. Grunberg, "Renormalization Group Improved Perturbative QCD," Phys. Lett. B 95, 70 (1980), [Phys. Lett. B 110, 501 (1982)].

25. J. D. Bjorken, "Applications of the Chiral $U(6) \times(6)$ Algebra of Current Densities," Phys. Rev. 148, 1467 (1966).

26. J. D. Bjorken, "Inelastic Scattering of Polarized Leptons from Polarized Nucleons," Phys. Rev. D 1, 1376 (1970).

27. A. Deur, V. Burkert, J. P. Chen and W. Korsch, "Experimental determination of the effective strong coupling constant," Phys. Lett. B 650, 244 (2007), [hep-ph/0509113].

28. A. Deur, V. Burkert, J. P. Chen and W. Korsch, "Determination of the effective strong coupling constant alpha(s,g(1))(Q**2) from CLAS spin structure function data," Phys. Lett. B 665, 349 (2008), [arXiv:0803.4119 [hep-ph]].

29. E. D. Bloom and F. J. Gilman, "Scaling, Duality, and the Behavior of Resonances in Inelastic electron-Proton Scattering," Phys. Rev. Lett. 25, 1140 (1970).

30. S. J. Brodsky and G. F. de Teramond, "Light-Front Holography and QCD Hadronization at the Amplitude Level," arXiv:0901.0770 [hep-ph].

31. C. Cruz-Santiago, P. Kotko and A. Stasto, "Recursion relations for multi-gluon offshell amplitudes on the light-front and Wilson lines," Nucl. Phys. B 895, 132 (2015), [arXiv:1503.02066 [hep-ph]].

32. J. P. Vary et al., "Hamiltonian light-front field theory in a basis function approach," Phys. Rev. C 81, 035205 (2010), [arXiv:0905.1411 [nucl-th]].

33. H. C. Pauli and S. J. Brodsky, "Solving Field Theory in One Space One Time Dimension," Phys. Rev. D 32, 1993 (1985). 
34. S. J. Brodsky and R. Shrock, "Condensates in Quantum Chromodynamics and the Cosmological Constant," Proc. Nat. Acad. Sci. 108, 45 (2011), [arXiv:0905.1151 [hepth]].

35. S. J. Brodsky, C. D. Roberts, R. Shrock and P. C. Tandy, "Confinement contains condensates," Phys. Rev. C 85, 065202 (2012), [arXiv:1202.2376 [nucl-th]].

36. A. V. Smirnov, V. A. Smirnov and M. Steinhauser, "Three-loop static potential," Phys. Rev. Lett. 104, 112002 (2010), [arXiv:0911.4742 [hep-ph]]. 